\title{
TEM/STEM Analysis of NiO Reduction to Ni during Annealing in $\mathrm{H}_{2}$ Atmosphere
}

\author{
Khachatur Manukyan $^{1}$, Suren Kharatyan ${ }^{2,3}$, Sergei Rouvimov ${ }^{4,5}$, and Alexander S.Mukasyan ${ }^{5,6}$ \\ ${ }^{1}$ Department of Physics, University of Notre Dame, Notre Dame, Indiana 46556, USA \\ ${ }^{2}$ Department of Chemistry, Yerevan State University, Yerevan, 0025, Armenia \\ ${ }^{3}$ Laboratory of Kinetics of SHS processes, Institute of Chemical Physics, Yerevan, 0014, Armenia \\ ${ }^{4}$ Department of Electrical Engineering, University of Notre Dame, Notre Dame, Indiana 46556, USA \\ ${ }^{5}$ Notre Dame Integrated Imaging Facility, University of Notre Dame, Notre Dame, Indiana 46556, USA \\ ${ }^{6}$ Department of Chemical and Bio-molecular Engineering, University of Notre Dame, Notre Dame, \\ Indiana 46556, USA
}

Nickel oxide $(\mathrm{NiO})$ reduction to $\mathrm{Ni}$ during annealing in hydrogen gas $(\mathrm{H} 2)$ atmosphere is of practical interest for catalytical applications [1] and for production of metallic Ni [2] particles. Recent in-situ TEM study [3] using monitoring of structure changes by EELS indicated that Ni reduction proceeds by $\mathrm{Ni}$ nucleation on $\mathrm{NiO}$ surface with further growth of $\mathrm{Ni}$ crystallites (often expitaxially) and the movement of growth front that is accompanied by the formation of pores within the $\mathrm{NiO}$ grains to accommodate the volume shrinkage associated with the reduction process. While this study was performed on $\mathrm{NiO}$ powder, the present study was focusing on structure evolution in bulk polycrystalline material.

The $\mathrm{Ni}$ wires were originally fully oxidized to $\mathrm{NiO}$. The $\mathrm{NiO}$ wires were reduced to $\mathrm{Ni}$ during annealing at $1230^{\circ} \mathrm{C}$ in hydrogen atmosphere (50 torr). The samples were analyzed in HRTEM and HAADF STEM modes using FEI Titan 80-300 electron microscope. The microscope was operated at $300 \mathrm{keV}$ and equipped with an Oxford Inca EDX detector. A TEM cross-sectional sample that included the NiO-Ni interface was prepared from the top surface by Focus Ion Beam (FIB) using FEI Helios SEM/FIB dual beam equipment.

As one can see from the cross sectional TEM and STEM images of Figure 1, the material is polycrystalline with relatively large grains (several um in diameter) that contains also large voids probably due to the volume shrinkage associated with the reduction process. EDS (Fig. 2a) indicated that $\mathrm{Ni}$ grains have almost no oxygen. The $\mathrm{Ni}$ grains have porous structure with small channels elongated almost parallel to some crystallographic directions (Fig. 2b) that may indicate on anisotropic oxidation during reduction process. The diameters of channels are ranging from 15 to $20 \mathrm{~nm}$, they form a net. The formation of the porous channels is associated with the volume shrinkage due to conversion of $\mathrm{NiO}$ to $\mathrm{Ni}$. During this process the crystalline structure of $\mathrm{Ni}$ is following crystalline lattice of $\mathrm{NiO}$ thus resembling the epitaxy as it was concluded earlier [3]. The oxygen from $\mathrm{NiO}$ is reacting with hydrogen which results in formation of the water molecules. The water vapor that is formed during high temperature reduction process can easily be transferred to the surface thus to provide a fast kinetic pathway for the reduction.

\section{References:}

[1] H. H. Kung, Transition metal oxides: surface chemistry and catalysis. (1989) Elsevier, New York

[2] T. Hidayat, MA Rhamdhani, E Jak, PC Hayes, Metall Mater Trans B 40 (2009)462

[3] Q. Jeangros et al, J Mater Sci 48 (2013) 2893-2907 


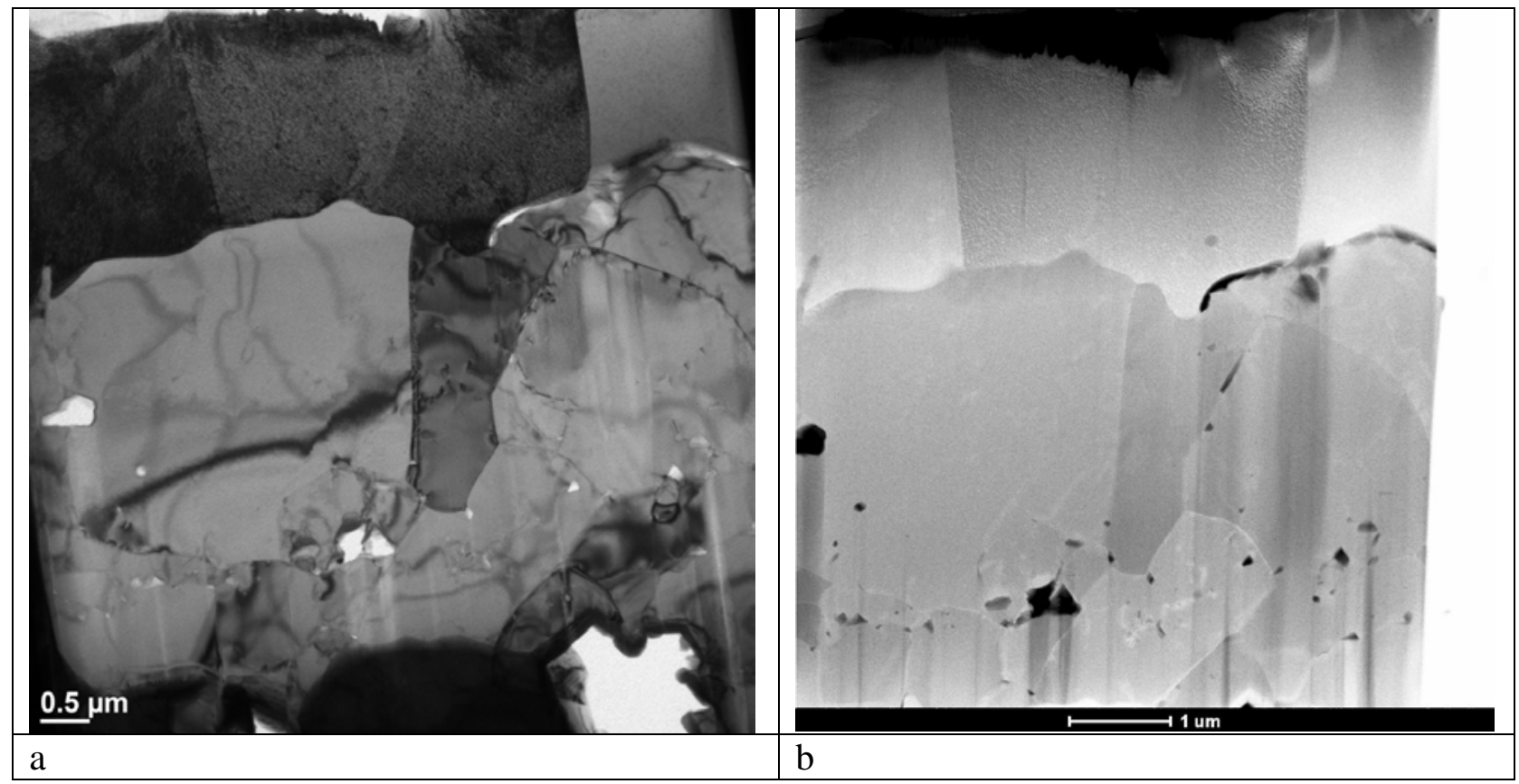

Figure 1. Cross-sectional TEM (a) and HDAAF STEM (b) images of Ni-NiO sample.

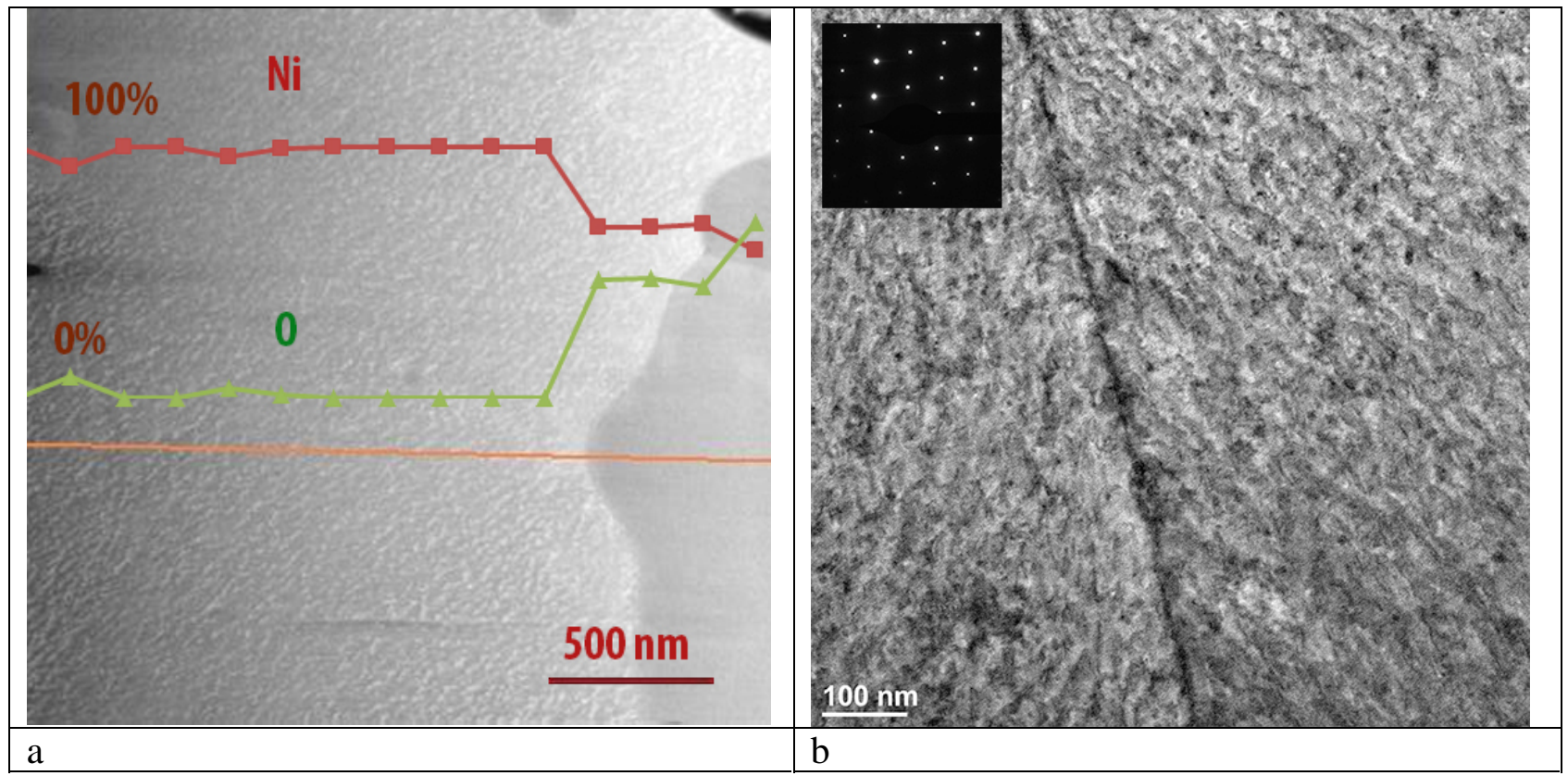

Figure 2. Cross-sectional HDAAF STEM (a) and TEM (b) images of Ni-NiO sample. Inserted are EDX profiles of $\mathrm{Ni}$ and $\mathrm{O}$ in the sample across the $\mathrm{Ni}-\mathrm{NiO}$ interface (a) and electron diffraction pattern (b). 